\title{
Combination of CT-guided hookwire localization and video-assisted thoracoscopic surgery for pulmonary nodular lesions: Analysis of 103 patients
}

\author{
WENTAO LI*, YING WANG* ${ }^{*}$ XINHONG HE, GUODONG LI, SHENGPING WANG, \\ LICHAO XU and ZHENG YUAN
}

Department of Radiology, Fudan University Shanghai Cancer Center, Shanghai 200032, P.R. China

Received March 20, 2012; Accepted June 28, 2012

DOI: $10.3892 / \mathrm{ol} .2012 .800$

\begin{abstract}
At present, there is no standardized method for the diagnosis and management of pulmonary nodular lesions (PNLs) smaller than $3 \mathrm{~cm}$. This study investigated the use of computed tomography (CT)-guided hookwire localization and video-assisted thoracoscopic surgery (VATS) for PNLs. A total of 103 patients undergoing CT-guided hookwire localization and VATS were enrolled, and 107 lesions were collected. We assessed the localization achievement ratio, complications rate, conversion thoracotomy rate, intraoperative dislodgement rate, pathological diagnosis rate, duration of surgery and average days of hospitalization. All 107 nodules from 103 patients were successfully localized (100\%), the asymptomatic pneumothorax rate was $36.9 \%$, the asymptomatic hemorrhage rate was $40.8 \%$ and the simultaneous pneumothorax and hemorrhage rate was $8.7 \%$. A conversion thoracotomy was required in $2(1.9 \%)$ patients and the intraoperative dislodgement rate was $2.9 \%$. The average time for localization was $11 \pm 4 \mathrm{~min}$, and the average times for wedge resection and lobectomies were $16 \pm 2$ and $95 \pm 30 \mathrm{~min}$, respectively. The mean hospitalization time following the surgery was $6 \pm 3$ days. All 107 nodules managed to achieve pathological diagnoses. A combination of CT-guided hookwire localization and VATS for PNL is a safe and efficient procedure of great clinical value.
\end{abstract}

Correspondence to: Professor Wentao Li or Dr Zheng Yuan, Department of Radiology, Fudan University Shanghai Cancer Center, 270 Dong An Road, Shanghai 200032, P.R. China

E-mail: liwentao98@126.com

E-mail: yuanzheng0404@163.com

*Contributed equally

Key words: pulmonary nodular lesions, ground-glass nodules, hookwire system, video-assisted thoracoscopic surgery

\section{Introduction}

Pulmonary nodular lesions (PNLs) are intraparenchymal lung lesions smaller than $3 \mathrm{~cm}$ in diameter, which are not associated with atelectasis or lymph node enlargement (1). According to the 2011 International Association for the Study of Lung Cancer (IASLC), American Thoracic Society (ATS) and European Respiratory Society (ERS) international multidisciplinary classification of lung adenocarcinoma, PNLs are classified as: a) a pure ground-glass nodule (pGGN) as a focal area of increased lung attenuation, which the margins of any normal structures, e.g., vessels, remain outlined; b) a solid nodule as a focal area of increased attenuation of such density that any normal structures, e.g., vessels, are completely obscured; c) part-solid nodule as a focal nodular opacity containing solid and ground-glass components (2). With the development of computed tomography $(\mathrm{CT})$, an increasing number of PNLs are detected. Since more than $50 \%$ of resected PNLs are related to malignancy, the requirement for rapid and definite histological diagnoses of PNLs has been stressed (3), in order for the treatment to occur as soon as possible. A transthoracic or transbronchial fine-needle biopsy may be considered in selected cases; however, the reported diagnostic yield for PNLs is rather low. An excisional biopsy of PNLs may be considered, but this requires invasive access, e.g., a thoracotomy. With the advent of video-assisted thoracoscopic surgery (VATS), a minimally invasive procedure has emerged that allows complete resection of PNLs with minimal morbidity. However, since the PNLs are small and/or far from the pleural surface this may limit VATS and render the intraoperative identification of PNLs difficult (4). Thus, failure to visualize or palpate PNLs has resulted in a conversion thoracotomy rate of up to $46 \%$ (5). Recently, a hookwire marking system has been proposed that involves preoperative CT-guided localization under local anesthesia and anchorage within the lesion. In this study, we describe the procedure and assess the value of CT-guided hookwire localization and VATS in 107 PNLs from 103 patients.

\section{Materials and methods}

Patient characteristics. Between January 2010 and December 2011, CT-guided hookwire localization and VATS were 
conducted on 107 PNLs from 103 patients who underwent CT examination at the Cancer Hospital of Fudan University (Shanghai, China). This study was composed of 45 males and 58 females, with a mean age of 54 years (range, 16-78 years). Of the 103 patients, 32 had a history of cancer. Patient characteristics are shown in Table I. Patients were informed of the risks associated with the procedure and written consent was obtained from all patients. The protocol in our study was approved by the institutional review board of Fudan University Shanghai Cancer Center, Shanghai, China.

Selection criterion and lesion characteristics. The selection criterion was based on at least one of the following CT findings: lesion diameter $\leq 10 \mathrm{~mm}$, distance from pleural surface $>5 \mathrm{~mm}$, pGGN or a lesion mostly comprised of GGNs. Lesion characteristics are shown in Table II. The diameter of the lesions ranged from 3.8 to $25.3 \mathrm{~mm}$ (mean, $13.0 \mathrm{~mm}$ ). The distance of the lesion from the pleural surface ranged from 1.6 to $40.3 \mathrm{~mm}$ (mean, $12.2 \mathrm{~mm}$ ). A total of $63(59 \%)$ and $44(41 \%)$ lesions displayed solid and GGNs, respectively. Among the 103 patients, 4 had 2 lesions to localize, which were situated in various lobes of the right lung.

CT-guided hookwire localization. A hookwire system is composed of a calibrated cannula (21-gauge, $10 \mathrm{~cm}$ long) and a $20-\mathrm{cm}$ long calibrated wire with a thorn. A CT scan was conducted to identify the location, size and shape of the lesion, as well as the correlation with the surrounding tissues. We then designed an optimal route by measuring the distance between the skin and the edge of the lesion and marking a puncture site. Following disinfection of the skin around the puncture site and local anesthesia, a narrow-ranged CT scan was conducted to ensure the correct puncture site, using the injection needle as a marker. The cannula needle housing the hookwire was inserted gradually through the chest wall and pulmonary parenchyma and placed as close as possible to the lesion (Fig. 1). When the outer cannula needle was withdrawn, the horn of the hookwire was released and a feeling of resistance emerged when the wire was pulled. A CT scan was repeated to confirm that the horn anchored the lesion, and to ensure that the existing complications, including pneumothorax (Fig. 2) and hemorrhage (Fig. 3), were present. The hookwire, extending outside the chest wall, was positioned carefully on the skin under gauze dressings. The patient was then transferred to the operating room for VATS. During this time, the images reconstructed following localization were uploaded for the thoracic surgeons to consult.

VATS. Video-assisted thoracoscopic resection of the lesion was conducted under general anesthesia using single lung ventilation via a double-lumen endobronchial tube. The procedure required 2 thoracic ports of $11.5 \mathrm{~mm}, 1$ for the thoracoscope, the other for the endoscopic stapler, and a $5.5 \mathrm{~mm}$ thoracic port for the lung forceps. The hookwire was raised during the procedure, and the lesion was sequentially resected (Fig. 4). The resected hookwire and lung tissue was packed into sterile gloves to prevent metastatic implantation of malignant disease and was withdrawn from the chest via an intercostal incision. All resected lung specimens were immediately sent for frozen-section examination. If the
Table I. Characteristics of 103 patients who underwent CT-guided hookwire localization and VATS.

\begin{tabular}{lr} 
Characteristic & No. of patients \\
\hline $\begin{array}{l}\text { Age (years) } \\
\leq 40\end{array}$ & \\
$>40$ & $11(10.7)$ \\
Gender & $92(89.3)$ \\
Male & \\
Female & $45(43.7)$ \\
Cancer history & $58(56.3)$ \\
Yes & \\
No & $32(31.1)$ \\
\hline
\end{tabular}

CT, computed tomography; VATS, video-assisted thoracoscopic surgery.

Table II. Characteristics of 107 PNLs from 103 patients who underwent CT-guided hookwire localization and VATS.

\begin{tabular}{lc}
\hline Characteristic & No. of patients $(\%)$ \\
\hline Location & \\
Upper lobe of right lung & $35(32.7)$ \\
Middle lobe of right lung & $6(5.6)$ \\
Lower lobe of right lung & $20(18.7)$ \\
Upper lobe of left lung & $24(22.4)$ \\
Lower lobe of left lung & $22(20.6)$ \\
Diameter (mm) & \\
$\leq 5$ & $3(2.8)$ \\
$5<\mathrm{d} \leq 10$ & $24(22.4)$ \\
$10<\mathrm{d}<30$ & $80(74.8)$ \\
Distance from pleural surface (mm) & \\
$\leq 5$ & $30(28.0)$ \\
$>5$ & $77(72.0)$ \\
Density & \\
GGN & $44(41.1)$ \\
Solid nodule & $63(58.9)$ \\
\hline
\end{tabular}

PNL, pulmonary nodular lesion; CT, computed tomography; VATS, video-assisted thoracoscopic surgery. d, diameter; GGN, ground-glass nodule.

pathological result was benign, a chest tube was inserted and VATS was conducted following bleeding and air leak exclusion. If the diagnosis was primary lung cancer, a lobectomy and lymphadenectomy were conducted, and if necessary, another thoracic incision was made to facilitate the subsequent thoracoscopic resection. If the result suggested a metastatic tumor following wedge resection, the procedure was terminated until a multidisciplinary treatment scheme was set up. In certain cases, we had to convert to a thoracotomy due to problems, including adhesion and hookwire dislodgement. 


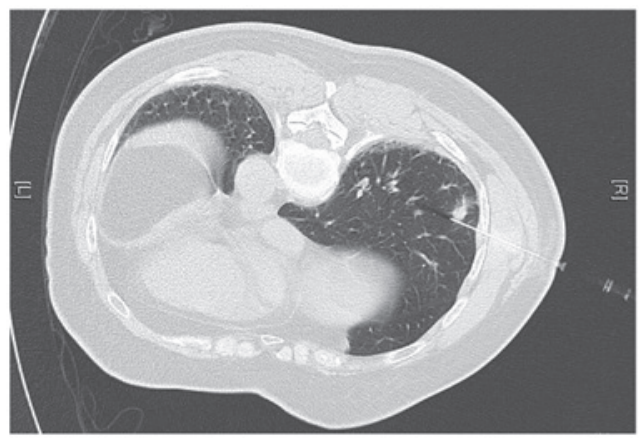

Figure 1. CT scan identifies a hookwire positioned on the edge of the lesion. $\mathrm{CT}$, computed tomography.

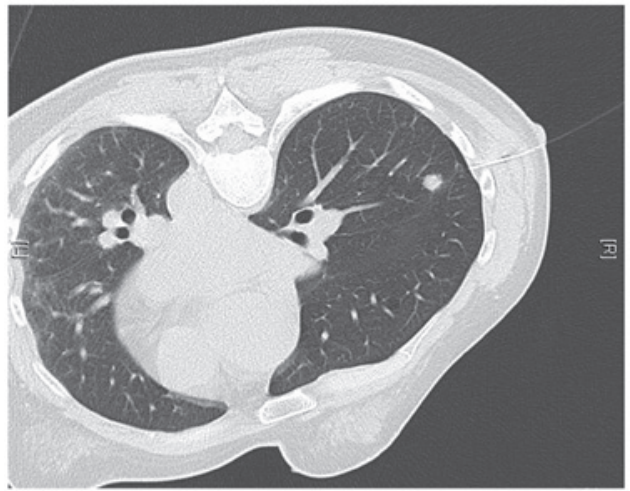

Figure 2. CT scan of minimal pneumothorax. CT, computed tomography.

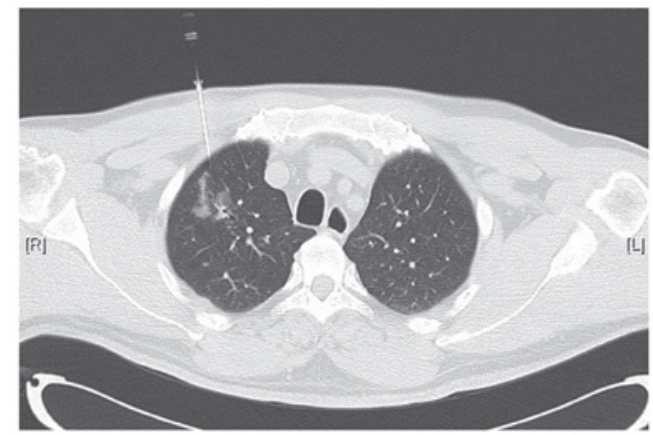

Figure 3. CT scan of asymptomatic hemorrhage around the lesion. CT, computed tomography.

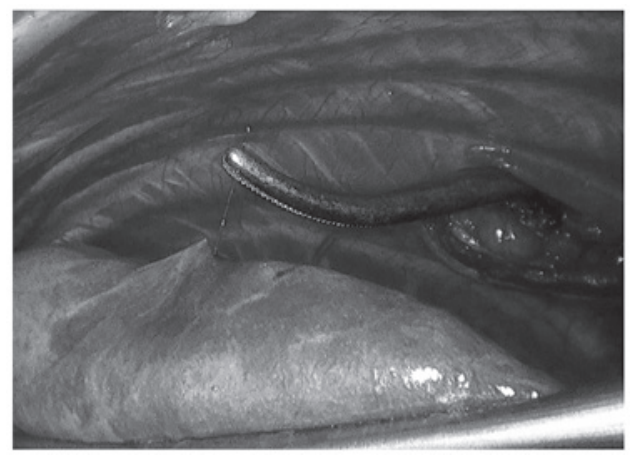

Figure 4. Lesion is raised and resected under VATS. VATS, video-assisted thoracoscopic surgery.
Table III. Characteristics of 103 patients who underwent CT-guided hookwire localization.

\begin{tabular}{lc}
\hline Characteristic & Value \\
\hline Posture, n (\%) & \\
Supination & $31(30.1)$ \\
Pronation & $45(43.7)$ \\
Left lateral decubitus & $15(14.6)$ \\
Right lateral decubitus & $12(11.6)$ \\
Complictions, n (\%) & \\
Pneumothorax & $38(36.9)$ \\
Hemorrhage & $42(40.8)$ \\
Pneumothorax and hemorrhage & $9(8.7)$ \\
Duration (min) & $6-27$ \\
Range & 11 \\
Mean & \\
Number of needle insertions or adjustments & $1-6$ \\
Range & 2 \\
Mean & \\
\hline
\end{tabular}

CT, computed tomography.

\section{Results}

All 107 lesions were localized successfully (100\%). The duration of localization, from the first CT scan to the last, ranged from 6 to $27 \mathrm{~min}$ (mean, $11 \mathrm{~min}$ ) and the number of needle insertions or adjustments ranged from 1 to 6 (mean, 2). The complications of CT-guided hookwire localization were asymptomatic pneumothorax, asymptomatic hemorrhage and simultaneous pneumothorax and hemorrhage, which were observed in $26.9,40.8$ and $8.7 \%$ of patients, respectively. VATS was conducted in all 103 patients. A total of 71 cases underwent wedge resection, including 43 benign lesions and 28 metastatic lesions. Among the other 32 patients, 28 received a lobectomy and lymphadenectomy and 4 abandoned surgery, including the two double primary cancer cases, due to their poor health condition or old age. The mean surgery time (excluding 20 min for frozen-section examination) for wedge resection and lobectomy was $16 \mathrm{~min}$ and $95 \mathrm{~min}$, respectively. Conversion thoracotomy was necessary in 2 patients; 1 PNL demonstrated strong adherence to the pleural surface, while the other, whose source was difficult to determine, demonstrated adhesion to the diaphragmatic muscle. Intraoperative dislodgement occurred in 3 patients, conversion thoracotomy occurred in 1 case, and the other 2 complications were identified and resected including observed bleeding from the area upon touching the lesion with a single finger. A total of 2 patients received a subsequent lobectomy, according to permanent-section and immunohistochemistry analysis, 1 week later. Postoperative complications of VATS, including 1 alveolar pleural fistula and 1 post-operative acataleptic thoracic hemorrhage, were both successfully managed by conservative treatment. The mean hospitalization time following the surgery was 
Table IV. Characteristics of 107 PNLs from 103 patients who underwent VATS.

\begin{tabular}{lc}
\hline Characteristic & Value \\
\hline Complictions, n (\%) & \\
Alveolar pleural fistula & $1(0.97)$ \\
Hemorrhage & $1(0.97)$ \\
Conversion thoracotomy & $2(1.94)$ \\
Dislodgement & $3(2.91)$ \\
Surgery time (min), mean \pm SD & \\
Wedge resection & $16 \pm 2$ \\
Lobectomy & $95 \pm 30$ \\
Re-lobectomy, n (\%) & $2(1.94)$ \\
Hospitalization time (days), mean \pm SD & $6 \pm 3$ \\
\hline
\end{tabular}

PNL, pulmonary nodular lesion; VATS, video-assisted thoracoscopic surgery.

Table V. Histological diagnosis of PNLs.

\begin{tabular}{lc}
\hline Characteristic & No. of patients $(\%)$ \\
\hline Benign & $43(40.2)$ \\
Atypical adenomatoid hyperplasia & 9 \\
Hamartoma & 7 \\
Non-caseating granuloma & 7 \\
Pulmonary tuberculosis & 2 \\
Inflammatory pseudotumor & 1 \\
Bronchial cyst & 1 \\
Lipoma & 1 \\
Lymphadenopathy & 1 \\
Other benign disease & 14 \\
Malignant & $64(59.8)$ \\
Adenocarcinoma & 43 \\
Atypical adenomatoid hyperplasia & 8 \\
with local cancerization & \\
Bronchioalveolar carcinoma & 3 \\
Leiomyosarcoma & 3 \\
Poorly differentiated cancer & 3 \\
Squamous cell carcinoma & 2 \\
Bronchioles clear cell carcinoma & 1 \\
Mucoepidermoid carcinoma & 1 \\
\hline
\end{tabular}

PNL, pulmonary nodular lesion.

$6 \pm 3$ days; however, the patient with acataleptic hemorrhage was discharged from hospital after 22 days. The characteristics of CT-guided hookwire localization and VATS are described in Tables III and IV. All 107 lesions managed to achieve pathological diagnoses. A total of $40.2 \%$ of the lesions were benign diseases, and detailed results are shown in Table V.

\section{Discussion}

The diagnosis and treatment of PNLs is a problem affecting radiologists and clinicians. PNLs have no typical imaging features and various kinds of biopsies, including percutaneous puncture biopsy and transbronchial needle biopsy, are much less invasive than surgery, but are less reliable for ruling out malignancy due to inadequate tissue sampling or biopsy failure (6). According to statistics, more than $50 \%$ of resected PNLs are related to malignancy; therefore, they should be considered potentially malignant until proven otherwise. For patients with PNLs, surgical resection is the ideal approach as it is diagnostic and therapeutic (7), and with regard to surgery, VATS may be the best option. With thoracoscopic technology development and surgical instrument refinement, VATS has been widely accepted for the resection of PNLs as it minimizes postoperative morbidity and saves as much lung tissue volume as possible. Furthermore, the survival rate is no less than open surgery in patients with stage Ia disease (8-10). However, certain patients require conversion to thoracotomy due to the change in anatomical position, the difficulty in distinguishing GGN with normal lung tissue and the inability to touch lesions far from the pleural surface following lung deflation. Therefore, it is necessary to conduct effective preoperative localization. At present, the most commonly used localization technique is CT-guided hookwire localization. Hookwire localization was originally applied in mammary glands. Then, with the advent of VATS, it was gradually used in localizing pulmonary lesions $(11,12)$, as well as lesions of the abdominal cavity, retroperitoneum and muscle, with the advantage of stronger anchorage and less invasion (13).

In this study, all 107 lesions from 103 patients were successfully localized. The mean duration of localization was $11 \pm 4 \mathrm{~min}$ and the mean surgery times for wedge resection and lobectomy were $16 \pm 2$ and $95 \pm 30$ min, respectively. With regard to surgery time, we did not make a comparison between localization with and without the CT-guided hookwire system prior to VATS. However, Ciriaco et al (14) reported that hookwire localization made VATS resection quicker, independent of whether it was conducted with preoperative localization or not. In this study, the average surgery time was $40 \pm 7$ and $75 \pm 12 \min (\mathrm{P}<0.001)$, and the average time of CT hookwire positioning was $20 \pm 10 \mathrm{~min}$.

In terms of complications, asymptomatic hemorrhage and minimal pneumothorax in the procedure of localization did not require clinical intervention. In the present study, 1 alveolar pleural fistula and 1 unidentified hemorrhage following VATS were successfully managed by conservative treatment. Dislodgement occurred in $3(2.91 \%)$ patients, and 1 case led to thoracotomy. The dislodgement rate in our study was consistent with a rate of $0.8-20 \%$ obtained from previous studies $(11,14,15)$. With the guidance of hookwire, lesions for frozen-section examination may be identified more quickly, which will greatly shorten the intraoperative waiting time. In this study, all 107 lesions achieved pathological diagnoses. Malignant lesions accounted for $59.8 \%$ of cases; this result is similar to $50-70 \%$, which was demonstrated in certain studies $(12,14,16,17)$, but significantly higher than $26 \%$, which was demonstrated in others (6). We suggest that this may be due to the benign diagnoses of certain patients in this study 
who were selected based on imaging results instead of pathological diagnoses, and the pathological diagnosis of patients lost during follow-up were not obtained. In our study, the probability of metastatic tumors in patients with PNLs and with cancer history was as high as $84.8 \%$.

The first principle of CT-guided localization is to obtain the shortest needle insertion route. It is agreed that the insertion of the hookwire should be vertical from the outside chest into the pleural surface. This not only indicates the definite and strong anchorage, but it also reduces the damage and complication incidence. The second principle of CT-guided localization is to avoid penetration through the lesion, in order for the hookwire to be placed infinitely near the lesion, but without transfixion. However, the option of touching or going through the lesion remains controversial. Certain researchers are concerned that penetrating through the lesion damages the intact capsula of the lesion and may prompt potential dissemination of the malignant tumor. However, Miyoshi et al suggested that no local recurrence of primary lung cancer was observed when lesions were penetrated (15). In our institute, we prefer the 'no penetrating' principle, which may maintain the integrity of the lesion. Additionally, penetrating solid nodules increases the difficulty of localization. The third principle of CT-guided localization is flexibility and cooperation. For certain lesions sheltered from bone structure, oblique needling insertion may be considered. For nodules close to the interlobar pleural, it is important to avoid damaging 2 lobes of the lung as much as possible. For lesions in the lower lung, which are easily influenced by respiration, the cooperation of the patient's respiration is of great importance. The final principle of CT-guided localization is to reduce the dislodgement rate. The hookwire should not be placed too shallow; it should be placed $2 \mathrm{~cm}$ beyond the edge of the lesion and once released, the free end of wire is cut off leaving a $2-3 \mathrm{~cm}$ tail.

From our experience and previous studies, we conclude that it is necessary to aggressively treat indeterminate PNLs. A combination of CT-guided hookwire localization and VATS for PNLs is a safe and effective procedure for accurate diagnosis and resection of indeterminate PNLs, with no associated mortality or significant morbidity.

\section{Acknowledgements}

This study was supported by the Science Technology Commission of Shanghai Municipality (0952nm03400, 11nm0504000).

\section{References}

1. Fraser RG, Sanders C, Barnes GT, et al: Digital imaging of the chest. Radiology 171: 297-307, 1989.

2. Travis WD, Brambilla E, Noguchi M, et al: International association for the study of lung cancer/american thoracic society/european respiratory society international multidisciplinary classification of lung adenocarcinoma. J Thorac Oncol 6: 244-285, 2011.

3. Shah PL, Singh S, Bower M, et al: The role of transbronchial fine needle aspiration in an integrated care pathway for the assessment of patients with suspected lung cancer. J Thorac Oncol 1: 324-327, 2006.

4. Ambrogi MC, Melfi F, Zirafa C, et al: Radio-guided thoracoscopic surgery (RGTS) of small pulmonary nodules. Surg Endosc 26: 914-919, 2012.

5. Suzuki K, Nagai K, Yoshida J, et al: Video-assisted thoracoscopic surgery for small indeterminate pulmonary nodules: indications for preoperative marking. Chest 115: 563-568, 1999.

6. Alzahouri K, Velten M, Arveux P, et al: Management of SPN in France. Pathways for definitive diagnosis of solitary pulmonary nodule: a multicentre study in 18 French districts. BMC Cancer 8: 93,2008

7. Tan BB, Flaherty KR, Kazerooni EA, et al: The solitary pulmonary nodule. Chest 123: 89S-96S, 2003.

8. Walker WS, Codispoti M, Soon SY, et al: Long-term outcomes following VATS lobectomy for non-small cell bronchogenic carcinoma. Eur J Cardiothorac Surg 23: 397-402, 2003.

9. Oda M, Matsumoto I, Tamura M, et al: Videoassisted thoracic surgery for clinical stage I lung cancer. Kyobu Geka 62: 281-284, 2009 (In Japanese).

10. Yan TD, Black D, Bannon PG and McCaughan BC: Systematic review and meta-analysis of randomized and nonrandomized trials on safety and efficacy of video-assisted thoracic surgery lobectomy for early-stage non-small-cell lung cancer. J Clin Oncol 27: 2553-2562, 2009.

11. Chen YR, Yeow KM, Lee JY, et al: CT-guided hook wire localization of subpleural lung lesions for video-assisted thoracoscopic surgery (VATS). J Formos Med Assoc 106: 911-918, 2007.

12. Pittet O, Christodoulou M, Pezzetta E, et al: Video-assisted thoracoscopic resection of a small pulmonary nodule after computed tomography-guided localization with a hook-wire system. Experience in 45 consecutive paients. World J Surg 31: 575-578, 2007.

13. Morrisen WB, Sanders TG, Parsons TW and Penrod BJ: Preoperative CT-guided hookwire needle localization of musculoskeletal lesions. AJR Am J Roentgenol 176: 1531-1533, 2001.

14. Ciriaco P, Negri G, Puglisi A, et al: Video-assisted thoracoscopic surgery for pulmonary nodules: rationale for preoperative computed tomography-guided hookwire localization. Eur J Cardiothorac Surg 25: 429-433, 2004.

15. Miyoshi K, Toyooka S, Gobara H, et al: Clinical outcomes of short hook wire and suture marking system in thoracoscopic resection for pulmonary nodules. Eur J Cardiothorac Surg 36: 378-382, 2009.

16. Chen S, Zhou J, Zhang J, et al: Video-assisted thoracoscopic solitary pulmonary nodule resection after CT-guided hookwire localization: 43 cases report and literature review. Surg Endosc 25: 1723-1729, 2011.

17. Hirai S, Hamanaka Y, Mitsui N, et al: Role of video-assisted thoracic surgery for the diagnosis of indeterminate pulmonary nodule. Ann Thorac Cardiovasc Surg 12: 388-392, 2006. 